\title{
Influencia en la proporción de residuos de construcción y la relación agua/cemento en la resistencia a la compresión y alabeo
}

\section{Influence on the proportion of construction waste and the water / cement ratio on the resistance to compression and warping}

\author{
Zadith N Garrido $\mathrm{C}^{1}$
}

\begin{abstract}
RESUMEN
El objetivo de este trabajo fue encontrar la influencia en la proporción de residuos de construcción y la relación agua / cemento en la resistencia a la compresión y alabeo en unidades de albañilería de 39 x 19 x $14 \mathrm{~cm}$. Para ello se obtuvo 27 bloquetas como muestra representativa mediante el tipo probabilístico para un modelo bifactorial. Procesada la información se presentó el resumen de los datos para su análisis correspondiente e interpretación, y para determinar la influencia de la proporción de residuos de construcción y la relación agua/cemento se utilizó el cálculo de los ensayos y análisis de laboratorio concluyendo que existe influencia significativa en la proporción de residuos de construcción, y decidiendo que las variables son dependientes. Los datos fueron desarrollados mediante ecuaciones y se procesaron con el software Statistix V. 8.0, para el estudio del cálculo trabajar al 35\%, con una relación agua/cemento de 0.50 . Los resultados de esta investigación constituyen una alternativa sustentable para el aprovechamiento de los materiales en la construcción de edificaciones, ya que reúne características tales como: ambientalmente benigna, socialmente aceptable y económicamente viable.
\end{abstract}

Palabras clave: residuos de construcción, bloquetas, concreto, resistencia a la compresión, alabeo.

\begin{abstract}
The purpose of this research was to find the influence on the proportion of construction waste and the water / cement ratio in the resistance to compression and warping in masonry units of $39 \times 19 \times 14$ $\mathrm{cm}$. For this, 27 blocks were obtained as a representative sample using the probabilistic type for a bifactorial model. Once the information was processed, the data summary was presented for its analysis and corresponding interpretation, and to calculate the influence of the proportion of construction waste and the water / cement ratio, the calculation of the Laboratory Tests and Analysis was used, concluding that there is Significant influence on the proportion of construction waste, and deciding that the variables are dependent, The data was developed using equations and processed with Statistix V. 8.0 software, to study the calculation work at $35 \%$, with a water / cement ratio.of 0.50 . The results of this research constitute a sustainable alternative for the use of materials in the construction of buildings, since it has characteristics such as: environmentally benign, socially acceptable and economically viable.
\end{abstract}

Keywords: construction waste, blocks, concrete, compression resistance, warping.

\footnotetext{
${ }^{1}$ Doctorado en Ciencia e Ingeniería, Universidad Nacional de Trujillo. Email: zadith.garrido@unj.edu.pe
} 


\section{INTRODUCCIÓN}

La excesiva demanda de viviendas unifamiliares en la ciudad de Tarapoto se debe a su desarrollo en los últimos 10 años. Algunos factores que han permitido este hecho, se debe a su ubicación estratégica dentro del marco regional, atraviesa la nueva y mejorada carretera Fernando Belaúnde Terry y además que el Aeropuerto Cadete FAP Guillermo del Castillo Paredes se encuentra en la ciudad, que es de gran importancia a nivel nacional y es punto de conexión principal hacia distintos departamentos de la selva peruana. Tarapoto pasó a ser de un pueblo de menos de 2000 habitantes desde su fundación en 1782, a dinamizador principal del sistema Urbano del departamento de San Martín que forma parte del Macro Sistema norte, de acuerdo al Plan Nacional de Desarrollo Urbano de 1990. Siendo su tipología económica la de centro urbano comercial, financiero, turístico y de servicios en general. Todo este movimiento crea un flujo demográfico que a la vez genera deficiencias de viviendas confortables y económicas que cubran este déficit urgente y primordial. Según el plan de desarrollo urbano de la ciudad, existen zonas de expansión urbana, las cuales están siendo ocupadas por invasiones. Dicha problemática nos lleva a focalizar al problema de la vivienda en esta ciudad de gran proyección a futuro. Acogiendo el tema sobre la influencia de la proporción de residuos de construcción y la relación agua/cemento en la resistencia a la compresión y alabeo en unidades de albañilería no portante, para todo tipo de edificaciones, que sea confortable y económica para una mejor calidad de vida, con un enfoque a la construcción de viviendas. La industria de la construcción es una de los mayores consumidores de recursos naturales como son los agregados gruesos y finos, utilizados en la producción de concreto, estos recursos son extraídos de canteras seleccionadas a las afueras de la ciudad donde la extracción es indiscriminada, altera el medio ambiente y en algún momento estos recursos se agotarán.

En este sentido, se propone crear unidades de albañilería con proporciones de residuos de construcción y ver su influencia en la resistencia a la compresión y alabeo (Vidarte, 2016, p.11). Los resultados de esta investigación constituyen una alternativa sustentable para el aprovechamiento de los materiales en la construcción de edificaciones, ya que reúne características tales como: ambientalmente benigna, socialmente aceptable y económicamente viable.

\section{MATERIALES Y MÉTODOS}

\section{Materiales}

Unidades de albañilería de concreto con residuos de construcción de dimensiones de 19 x 39 x 14 cm. huecos no portantes. 
Influencia de la proporción de residuos de construcción y la relación agua/cemento

Población: La población estará determinada por las unidades de albañilería con residuos de construcción. Muestra: 27 Unidades de albañilería de dimensiones 19x39x14 cm.

Muestreo: La investigación es del tipo probabilístico para un modelo bifactorial.

\section{Equipos:}

Mallas de granulometría del Laboratorio de la Universidad Nacional de San Martín. Equipo de compresión de unidades de albañilería.

Balanza digital.

Dos cuñas de acero graduadas a medio milímetro para alabeo.

Para muestras:

Exactitud $45 \mathrm{~g}$ o dentro de $3 \%$ de peso.

Varilla o vibrador.

Varilla de Ø 5/8” (16 mm) x 24” (600mm).

Recipiente metálico (molde).

Capacidad de acuerdo a TM.

Placa de Enrasado Espesor $\geq 1 / 4 "(6 \mathrm{~mm})$, Largo y ancho Ø recipiente +2 ".

Mazo de goma.

\section{Métodos}

En la Tabla 1, se describen los instrumentos además de los fuentes o informantes respectivos.

Tabla 1. Relación de instrumentos empleados

\begin{tabular}{ll}
\hline Instrumentos & Fuentes o informantes \\
\hline Guía de observación. & Materia prima. \\
Fichas de recolección de datos & Zona de estudio. \\
Ficha bibliográfica & Reglamento Nacional \\
& de Edificaciones. \\
\hline
\end{tabular}

El número de repeticiones es de 3 según Mongomeri, Gutiérrez de la Bara. Las muestras como son homogéneas serán 3 unidades por el número de veces repetidas.

\section{La matriz de datos}

Tabla de datos para resistencia a la compresión y alabeo $\mathrm{N}=$ a.b.r $=3$ × 3 × $3=27$ 


\section{Análisis granulométrico de los agregados}

Peso unitario compactado de los agregados (fino y grueso) Peso específico de los agregados (fino y grueso).

Contenido de humedad y porcentaje de absorción de los agregados (fino y grueso) Perfil y textura de los agregados.

Tipo y marca del cemento.

Peso específico del cemento.

Relaciones entre resistencia y la relación agua/cemento, para combinaciones posibles de cemento y agregados.

Residuos de construcción de edificaciones triturados libre de acero.

El espécimen debe ser pesado inmediatamente después de que se reciben y se marcan. Se toman las dimensiones de longitud, altura, ancho y espesores. Se ensayan a compresión tres especímenes, que deben ser coronados.

Se debe alinear el espécimen en la máquina de ensayo de manera que su centroide coincida con el punto de aplicación de la carga. El espécimen se debe ensayar con dos placas que estén en contacto con el espécimen y permitan la distribución uniforme de la carga.

Se registra la carga máxima alcanzada por el espécimen. La resistencia a la compresión se calcula dividiendo la carga máxima entre el área neta.

La muestra estará constituida por ladrillos enteros obtenidos según la Norma ITINTEC 331.019. Pueden usarse los mismos ladrillos usados en la determinación de dimensiones para la resistencia a la compresión. Según el alabeo se presenta como concavidad o convexidad, seguir el procedimiento que para cada caso se detalla a continuación en las dos caras mayores del ladrillo. La Medición de concavidad, se coloca el borde recto de la regla ya sea longitudinalmente o sobre una diagonal de una de las caras mayores del ladrillo. Se introduce la cuña en el punto correspondiente a la flecha máxima. Se efectúa la lectura con la precisión de $1 \mathrm{~mm}$ y se registra el valor obtenido. Medición de convexidad, se emplea alternativamente uno de los procedimientos siguientes:

a) Se coloca al borde recto de la regla sea sobre una diagonal o bien sobre dos aristas opuestas de una 
de las caras mayores del ladrillo. Se introduce en cada vértice una cuña y se busca el punto de apoyo de la regla sobre la diagonal, para el cual en ambas cuñas se obtenga la misma medida.

b) Se apoya el ladrillo por la cara a medir sobre una superficie plana, se introduce cada una de las cuñas en dos vértices opuestos diagonalmente o en dos aristas, buscando el punto para el cual en ambas cuñas se obtenga la misma medida.

Se indica el promedio de los valores correspondientes a concavidad y/o convexidad obtenidos en milímetros enteros. Probetas o bloques de concreto.

Las probetas no serán ensayadas si cualquier diámetro individual de un cilindro difiere de cualquier otro diámetro del mismo por más del $2 \%$.

\section{Procedimiento}

Los ensayos a compresión de probetas del curado húmedo serán hechos tan pronto como sea práctico luego de retirarlos del almacenaje húmedo. Los cilindros serán protegidos de perdida de humedad por cualquier método conveniente durante el periodo entre el retiro del almacenaje de humedad y el ensayo. Los cilindros serán ensayados en condición húmedos. Todos los cilindros de ensayo para una determinada edad de ensayo serán fracturados dentro del tiempo permisible de tolerancia prescrita.

Colocar el bloque de rotura inferior, sobre el cabezal de la máquina de ensayo. El bloque de rotura superior directamente bajo la rótula del cabezal. Limpiar las caras de contacto de los bloques superior e inferior y las de la probeta de ensayo y colocar el cilindro sobre el bloque inferior de rotura. Cuidadosamente alinear los ejes de la probeta con el centro de empuje de la rótula del bloque asentado. Antes de ensayar la probeta verificar que el indicador de carga este en cero, ajustar el indicador.

La carga será aplicada a una velocidad de movimiento correspondiente a una velocidad de esfuerzo sobre la probeta de $0.25 \pm 0.05 \mathrm{MPa} / \mathrm{s}$. La velocidad de movimiento diseñada será mantenida al menos durante la mitad final de la fase de carga anticipada.

No hacer ajustes en la velocidad de movimiento cuando la carga final está siendo aplicada y la velocidad de esfuerzo decrece debido a la fractura del espécimen.

La resistencia a la compresión se calcula dividiendo la fuerza aplicada entre el área de la probeta ensayada, ver Tabla 2 donde se listan los instrumentos empleados para este fin. 
Tabla 2. Relación de instrumentos empleados para determinar la resistencia

\begin{tabular}{cc}
\hline Métodos & Técnicas \\
\hline Observación & Observación \\
Observación & Análisis de documentos \\
$\begin{array}{c}\text { Experimentación: de las respuestas a } \\
\text { reacciones específicas } \\
\text { Observación }\end{array}$ & Aparatos físicos \\
\hline
\end{tabular}

\section{RESULTADOS}

Obteniendo el cálculo respectivo para la determinación de los pesos, para después proceder a realizar 15 especímenes de $6 \times 12$ pulgadas de las cuales se han distribuido en 5 por patrón valorados y calculados mediante la rotura realizando las relaciones entre $0.50,0.60$ y 0.70 una vez obtenido el resultado se ha procedido a la elaboración de 27 unidades de albañilería de 39 × 19 × 14 con las mezclas respectivas, terminado los especímenes se procedió a la rotura de la misma, obteniendo los resultados que se muestran en el presente estudio, ver Tabla 3.

Tabla 3. Porcentaje de residuos de construcción en relación a \% de agregados

\begin{tabular}{|c|c|c|c|c|}
\hline \multirow[t]{4}{*}{$\%$} & $\begin{array}{ll}\text { Residuos de } \\
\text { construcción }\end{array}$ & 0.50 & 0.60 & 0.70 \\
\hline & $30 \%$ & 117.22 & 113.19 & 107.70 \\
\hline & $40 \%$ & 109.43 & 106.59 & 97.32 \\
\hline & $50 \%$ & 101.97 & 98.01 & 93.41 \\
\hline
\end{tabular}

\section{Análisis de la variable resistencia}

Tabla 4. Análisis de varianza para la variable resistencia

\begin{tabular}{|c|c|c|c|c|c|}
\hline Fuente de variación & $\begin{array}{l}\text { Grados de } \\
\text { libertad }\end{array}$ & $\begin{array}{l}\text { Suma } \\
\text { cuadrado }\end{array}$ & $\begin{array}{l}\text { Cuadrados } \\
\text { medios }\end{array}$ & F calculado & $\begin{array}{l}\text { Valor de } \\
\text { Probabilidad }\end{array}$ \\
\hline RESIDUOS & 2 & 8938.6 & 4469.30 & 282457 & 0.0000 \\
\hline $\mathrm{AC}$ & 2 & 4140.9 & 2070.45 & 130851 & 0.0000 \\
\hline RESIDUOS*AC & 4 & 171.5 & 42.87 & 2709.09 & 0.0000 \\
\hline Error & & 45 & 0.7 & 0.02 & \\
\hline Total & & 53 & 13251.7 & & \\
\hline Media Total & 220.51 & $\begin{array}{l}\text { Coeficiente de } \\
\text { Variación }\end{array}$ & 0.06 & & \\
\hline
\end{tabular}


Influencia de la proporción de residuos de construcción y la relación agua/cemento

Existen diferencias altamente significativas entre los residuos con respecto a la resistencia a la compresión.

Existen diferencias altamente significativas entre la relación agua / cemento con respecto a la resistencia a la compresión.

La interacción RESIDUOS*AC resultó altamente significativa, lo que indica que los factores son dependientes, ver Tabla 4.

Tabla 5. Prueba de Resistencia por residuos

\section{Tukey HSD Comparaciones múltiples Prueba de Resistencia por Residuos}

\begin{tabular}{cccc}
\hline RESIDUOS & Media & \multicolumn{2}{c}{ Grupos Homogéneos } \\
& 1 & 236.82 & A \\
& 2 & 219.34 & B \\
& 3 & 205.37 & C
\end{tabular}

Nivel de significancia 0.05 y error estándar para la comparación 0.0419

Valor crítico de Q 3.428 Valor crítico para la comparación 0.1016

Termino del error usado: Error, 45 grados de libertad.

Todas las 3 medias presentan diferencias significativas.

El residuo 1 es el que genera un mayor valor de resistencia a la compresión, Tabla 5.

Tabla 6. Prueba de Resistencia por agua / cemento

Tukey HSD Comparaciones múltiples Prueba de Resistencia por agua cemento

\begin{tabular}{ccc}
\hline AC & Media & \multicolumn{2}{l}{ Grupos Homogéneos } \\
\hline 1 & 230.04 & A \\
2 & 222.59 & B \\
3 & 208.90 & C \\
\hline
\end{tabular}

Nivel de significancia 0.05 y error estándar para la comparación 0.0419

Valor crítico de Q 3.428 Valor crítico para la comparación 0.1016, ver Tabla 6. 


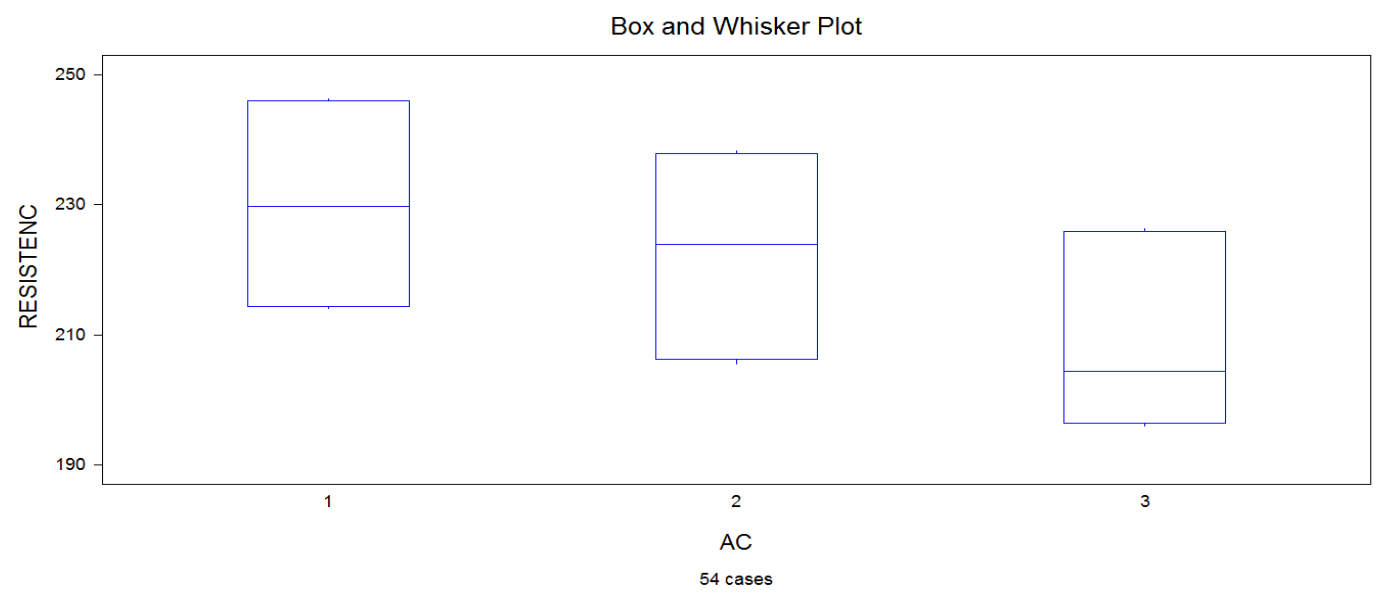

Figura 1. Relación de Agua / cemento para determinar su resistencia

Termino del error usado: Error, 45 grados de libertad.

Todas las 3 medias presentan diferencias significativas.

La relación agua / cemento 1 es el que genera un mayor valor de resistencia a la compresión, como se puede apreciar en la Figura 1.

\section{Análisis de variable alabeo}

Tabla 7. Análisis de varianza para la variable Alabeo

\begin{tabular}{|c|c|c|c|c|c|c|}
\hline Fuente de variación & $\begin{array}{l}\text { Grados de } \\
\text { libertad }\end{array}$ & $\begin{array}{l}\text { Suma } \\
\text { cuadrado }\end{array}$ & de & $\begin{array}{l}\text { Cuadrados } \\
\text { medios }\end{array}$ & F calculado & $\begin{array}{l}\text { Valor de } \\
\text { Probabilidad }\end{array}$ \\
\hline RESIDUOS & 2 & 0.25926 & & 0.12963 & 1.84 & 0.1702 \\
\hline $\mathrm{AC}$ & 2 & 0.14815 & & 0.07407 & 1.05 & 0.3575 \\
\hline RESIDUOS*AC & 4 & 0.01852 & & 0.00463 & 0.07 & 0.9918 \\
\hline Error & & & & 45 & 3.16667 & 0.07037 \\
\hline Total & & & & 53 & 3.59259 & \\
\hline
\end{tabular}

Media Total 1.8704

Coeficiente de Variación 14.18

Los residuos no tienen un efecto significativo en el alabeo, es decir, se comportan de la misma manera. La relación agua / cemento no tienen un efecto significativo en el alabeo, es decir, se comportan de la misma manera.

En la Tabla 7, la interacción RESIDUOS*AC es no significativa, lo que indica que los factores son independientes.

En las Figura 2, Figura 3 y Figura 4 respectivamente, mediante un diagrama de caja y bigotes se muestra la relación de residuos de construcción para determinar el alabeo, relación de residuos de construcción para determinar su resistencia y relación de agua/cemento para determinar el alabeo 
Influencia de la proporción de residuos de construcción y la relación agua/cemento

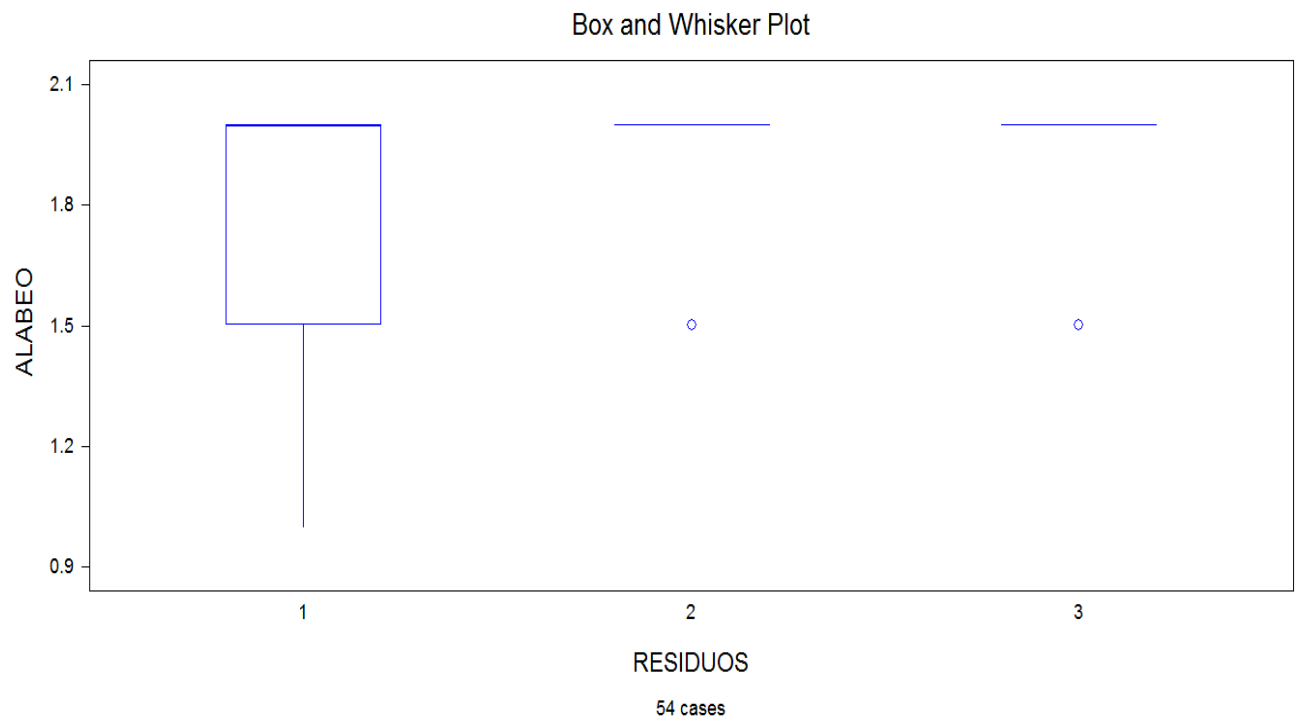

Figura 2. Relación de residuos de construcción para determinar el alabeo

Box and Whisker Plot

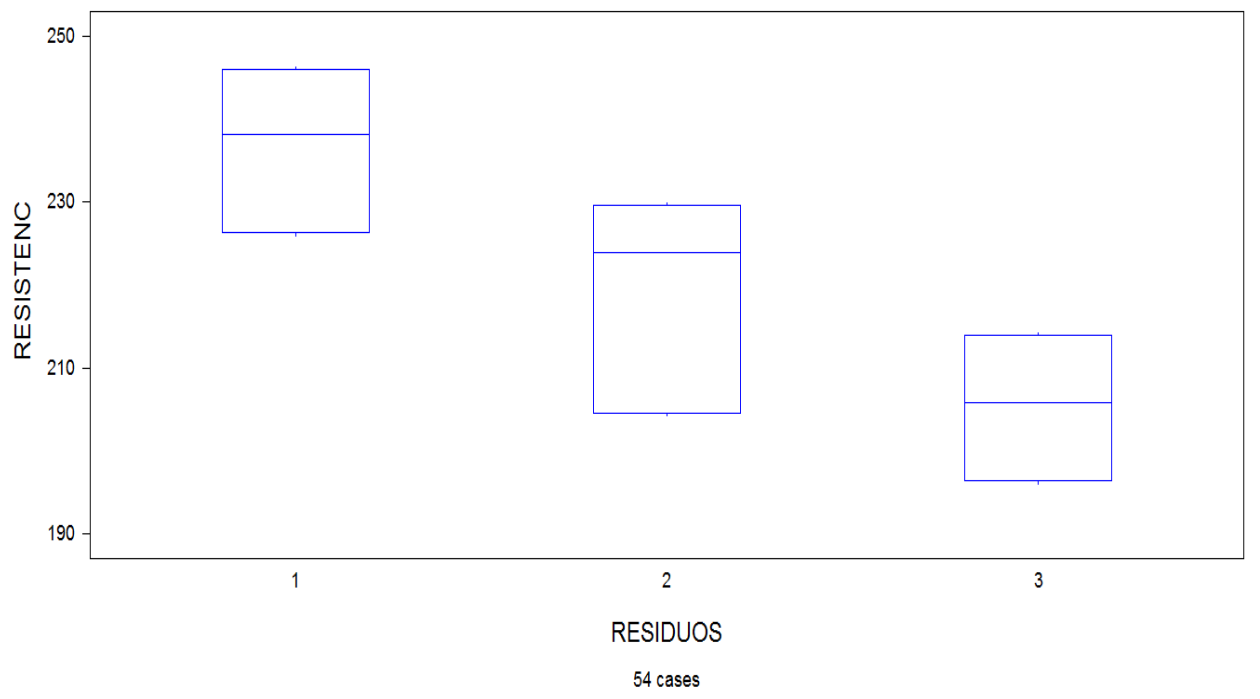

Figura 3. Relación de residuos de construcción para determinar su resistencia 


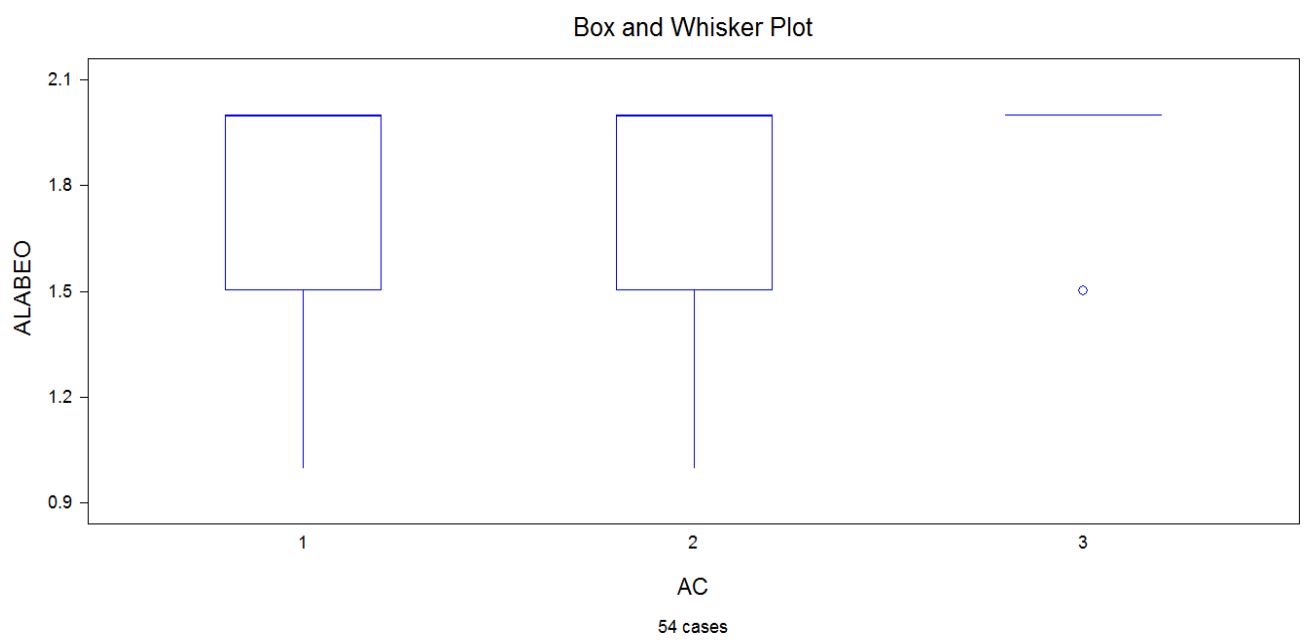

Figura 4. Relación de agua/cemento para determinar el alabeo

\section{DISCUSIÓN}

Al ser este trabajo de tipo básica aplicada, se analizó la influencia de las variables de estudio de acuerdo a los objetivos establecidos, donde se obtuvo los resultados que más transcendieron:

Recolectado las muestras del Centro de acopio de los agregados gruesos y chancado de tamaño máximo nominal de 1 pulgada del Rio Huallaga más el agregado fino del Rio Cumbaza se procedió a la selección, colección y transporte de las muestras a los laboratorios de la Universidad Nacional de San Martin para llevar a cabo la determinación de las propiedades físicas, mecánicas y químicas de los mencionados agregados luego se procedió a recolectar los residuos de construcción de las diversas obras existentes en la Ciudad de Tarapoto, para posteriormente ser preparados y procesados mediante la rotura de los elementos estructurales donde se calculó el módulo de fineza pasando el tamiz $\mathrm{N}^{\circ} 4$ y lo que retiene el tamiz $\mathrm{N}^{\circ} 200$. Una vez ejecutado el procedimiento se determinó las propiedades físicas, mecánicas y químicas del material obtenido entre los agregados y los residuos de construcción, seguidamente se procedió al diseño de mezclas, como el ensayo resistencia a la compresión con un fc $210 \mathrm{Kg} / \mathrm{cm}^{2}$ del agregado grueso del Rio Huallaga y agregado fino Rio Cumbaza, inmediatamente se realizó los diseños de mezclas con los porcentajes de residuos de construcción al 30, 40 y $50 \%$. Estudios relacionados con los resultados obtenidos en la influencia de la proporción de residuos de construcción y la relación agua/cemento en la resistencia a la compresión y alabeo en unidades de albañilería no portante, se encuentra en Guzmán (2016), donde en sus resultados de investigación menciona su producción industrial y comercialización podrían ayudar a reciclar sobrantes de los miles de obras civiles que se hacen en nuestro país.

También contribuiría a mitigar ciertos problemas ambientales como la sobreexplotación de bancos de 
materiales vírgenes, además de la contaminación atmosférica, pues el secado del ladrillo ecológico no genera emisiones como sucede en el caso de la cocción de tabique en hornos: en el Perú, 16 mil 953 productores artesanales queman diferentes combustibles que producen gases de efecto invernadero.

Así mismo Veliz, J. Zambrano, E. Rivera, R. (2013). En su tema de investigación Reciclaje de residuos de construcción en la producción de bloques en la ciudad de Portoviejo afirman que el contenido de los residuos influye en las características del bloque principalmente en la resistencia. Siendo el contenido de ladrillo el más influyente, ya que a menor contenido se eleva la resistencia.

\section{Análisis de la variable resistencia}

Mamani, F. (2015). Producción de agregados reciclados de los residuos de la construcción y demolición para la producción de concretos hidráulicos en la ciudad de Juliaca. Universidad Andina Néstor Cáceres Velásquez. Perú. Los resultados obtenidos de la resistencia a la compresión de concretos con agregados reciclados en sus diferentes combinaciones demuestran que es posible usar estos concretos en elementos estructurales, la combinación 01 alcanzó al 81.73\%, la combinación 02 alcanzó al 84.51\% y la combinación 03 alcanzó al 107.22\%.

En la presente investigación existen diferencias altamente significativas entre los residuos con respecto a la resistencia a la compresión.

Existen diferencias altamente significativas entre la relación agua / cemento con respecto a la resistencia a la compresión.

La interacción RESIDUOS*AC resultó altamente significativa, lo que indica que los factores son dependientes.

El residuo 1 es el que genera un mayor valor de resistencia a la compresión.

La relación agua / cemento 1 es el que genera un mayor valor de resistencia a la compresión.

Hahn. (2001) En Holanda y Dinamarca entre un 80 y un $85 \%$ de los residuos de la construcción vienen del hormigón y albañilería. Sólo el hormigón cubre un 30 - 40\%. Esto puede compararse con la situación en Kuwait, donde los escombros de hormigón representan aproximadamente el 30\% de los deshechos de demolición. Basado en los porcentajes que reflejan una rigurosa predicción sobre la producción anual de residuos de escombros de hormigón en los países de la comunidad Europea da una cifra entre los 30 y 50 millones de toneladas.

En la presente investigación se utiliza el 30\%, 40\% y 50\% de los residuos de construcción para su aprovechamiento como alternativa de uso de materiales en construcción de unidades de albañilería en edificaciones. 


\section{Análisis de variable alabeo}

Los residuos no tienen un efecto significativo en el alabeo, es decir, se comportan de la misma manera. La relación agua / cemento no tienen un efecto significativo en el alabeo, es decir, se comportan de la misma manera. La interacción RESIDUOS*AC es no significativa, lo que indica que los factores son independientes.

\section{CONCLUSIONES}

Luego de presentar los resultados, interpretarlos y discutirlos, se concluye que: Se determinó al 30\% de residuos de construcción con relación agua/cemento de 0.50 se obtuvo un resultado de $117.22 \%$ equivalente a $246.17 \mathrm{Kg} / \mathrm{cm}^{2}$, con relación agua/cemento de 0.60 se obtuvo un resultado de $113.19 \%$ equivalente a $238.12 \mathrm{Kg} / \mathrm{cm}^{2}$ y con relación agua/cemento de 0.70 se obtuvo un resultado de $107.70 \%$ equivalente a $226.17 \mathrm{Kg} / \mathrm{cm}^{2}$.

Se determinó al $40 \%$ de los residuos de construcción con relación agua/cemento de 0.50 se obtuvo un resultado de $109.43 \%$ equivalente a $229.81 \mathrm{Kg} / \mathrm{cm}^{2}$, con relación agua/cemento de 0.60 se obtuvo un resultado de $106.59 \%$ equivalente a $223.84 \mathrm{Kg} / \mathrm{cm}^{2}$ y con relación agua/cemento de 0.70 se obtuvo un resultado de $97.32 \%$ equivalente a $204.37 \mathrm{Kg} / \mathrm{cm}^{2}$.

Se determinó al 50\% de los residuos de construcción con relación agua/cemento de 0.50 se obtuvo un resultado de $101.97 \%$ equivalente a $214.15 \mathrm{Kg} / \mathrm{cm}^{2}$ con relación agua/cemento de 0.60 se obtuvo un resultado de $98.01 \%$ equivalente a $205.81 \mathrm{Kg} / \mathrm{cm}^{2}$ y con relación agua/cemento de 0.70 se obtuvo un resultado de $93.41 \%$ equivalente a $196.15 \mathrm{Kg} / \mathrm{cm}^{2}$.

\section{REFERENCIAS BIBLIOGRÁFICAS}

Vidarte, 2016. P 11. Unidades de albañilería con proporciones de residuos de construcción y ver su influencia en la resistencia a la compresión y alabeo.

Hahn, N. (2001). Producción de residuos de construcción y reciclaje. España: Madrid.

Veliz, J. Zambrano, E. Rivera, R. (2013). Reciclaje de residuos de construcción en la producción de bloques en la ciudad de Portoviejo. Universidad Técnica de Manabí. Ecuador.

Mamani, F. (2015). Producción de agregados reciclados de los residuos de la construcción y demolición para la producción de concretos hidráulicos en la ciudad de Juliaca. Universidad Andina Néstor Cáceres Velásquez. Perú.

Guzmán, F. Instituto de Ingeniería. (2016) El ladrillo ecológico basado en residuos de construcciones. 